\title{
LAS REDES DE TELECOMUNICACIONES DE CABLE HISTÓRICO: REALIDAD Y TENDENCIAS
}

\section{THE CABLE TELECOMMUNICATIONS NETWORKS HISTORICAL FACT AND TRENDS}

\section{AUTOR}

\section{Joan Francesc Fondevila Gascón}

Profesor del Doctorado en Sociedad de la Información y el Conocimiento y director del CECABLE (Centro de Estudios sobre el Cable). Universitat Oberta de Catalunya (UOC) y CECABLE. (España).

jfondevilag@uoc.edu

\section{RESUMEN}

Los operadores de cable histórico en España han actuado como dinamizadores de las comunicaciones durante décadas, garantizando la recepción de la televisión en numerosos municipios. El caso de Cataluña permite analizar el peso territorial y de negocio de las empresas de cable local. Los cambios legislativos y la consolidación y la opción de ofrecer los servicios de Internet y telefonía están provocando en esas redes locales de telecomunicaciones por cable un escenario de cambio, en el que se observan diversas opciones de adaptación. La absorción por parte de los grandes operadores, la desaparición o la continuidad en solitario son los posibles caminos de futuro para estas redes.

\section{PALABRAS CLAVE}

Cable - Telecomunicaciones - Sociedad de la Información y el Conocimiento 


\section{ABSTRACT}

The historical cable operators in Spain have acted revitalizing the communications during decades, guaranteeing the receipt of the television in a lot of municipalities. The case of Catalonia allows to analyze the business and territorial weight of the local cable companies. The legislative changes and the consolidation and the option to offer the Internet and telephony services are provoking in these local cable telecommunications networks a scene of change, in that diverse options of adjustment are observed. The absorption on the part of the big operators, the disappearance or the continuity are the possible ways of future for these networks.

\section{KEY WORDS}

Cable - Telecommunications -Information and Knowledge Society

\section{ÍNDICE}

1. El cable histórico en el marco de la Sociedad de la Información y el Conocimiento

2. La realidad del desarrollo del cable

2.1 La penetración del cable y la amenaza del ADSL

2.2 Obstáculos a superar para el aumento de la penetración del cable

3. El caso de Cataluña: la transición para el cable histórico

3.1 El peso cuantitativo del cable local

3.2 Los condicionantes legislativos del cable histórico

4. Conclusión

5. Bibliografía 


\section{El cable histórico en el marco de la Sociedad de la Información y el Conocimiento}

El sector de las redes de telecomunicación por cable se halla en los inicios del siglo XXI en una encrucijada, especialmente las redes tradicionales ubicadas en general en pequeños municipios. Estas empresas de cable son conocidas como operadores de cable histórico, ya que fueron pioneras en aparecer y durante años funcionaron alegalmente a raíz de la inexistencia de una legislación que las amparase. Las redes adjudicatarias de los concursos surgidos de la Ley 42/1995, de 22 de diciembre, de las Telecomunicaciones por Cable han visto ralentizado su ritmo de implantación por diversos motivos (multiplicación de las ofertas tecnológicas, dificultades en la obtención de permisos por parte de las Administraciones públicas involucradas 0 moderación en las inversiones).

El impacto de las redes de banda ancha sensibiliza a los estamentos europeos desde hace una década (Comisión Europea, 1994). De hecho, el marco teórico de la Sociedad de la Información y el Conocimiento está recibiendo aportes desde sectores adyacentes como las ciencias de la computación (Gates, 1995) o la sociología (Castells, 1997), que tratan de interpretar la sociedad digital o la sociedad red en la que nos hallamos inmersos.

La tarea de comprender la realidad y las tendencias de los operadores de cable histórico enlaza con el fenómeno de la televisión local (Garriga, 1985; Farias y Multigner, 1995; Calvo Charro, 1997), en una dinámica del audiovisual mutante y enfocada a negocio (Castilla, 1989; Regourd, 1992; Bustamante, 1999). El total de kilómetros de fibra óptica extendida en España y en Cataluña es muy diverso en función de las fuentes (López-Escobar y Bertrand, 1986; Junta de Castilla y León, 1995; Fondevila Gascón, 2003). La extensión de la red de telecomunicaciones por cable y de banda ancha es de complejo diagnóstico, en parte porque el cambiante concepto de banda ancha no está consensuado por la comunidad científica. Existen 
aproximaciones mediante leyes telecomunicativas (Gilder, 2002), como las de Moore o Metcalfe, pero la percepción es que el concepto es maleable, flexible, adaptable a la evolución de la demanda de los usuarios. Además, se está configurando una alternativa a la red de cable como puerta de acceso a la banda ancha: el ADSL (Asymmetrical Digital Subscriber Line), que se puede considerar banda estrecha ampliada. En todo caso, la penetración de la banda ancha es un indicador correcto para analizar la evolución en el camino hacia una sociedad pandigitalizada y en la que surgen propuestas sobre modelos de negocio alrededor de un sector con grandes posibilidad de proyección (Marcus, 1986; De Fontenay et altri, 1990) y estudiado desde la perspectiva teórica y metodológica de la economía política de la información (Mosco y Wasko, 1988).

\section{La realidad del desarrollo del cable}

\subsection{La penetración del cable y la amenaza del ADSL}

Teniendo en cuenta que los índices de acceso a Internet con banda ancha son poco significativos (Fondevila Gascón, 2004a), las cifras catalanas en este momento son aceptables, pese a situarse a cierta distancia de los países europeos, americanos y asiáticos más avanzados. En 2000, Cataluña contaba con 49,3 líneas por 100 habitantes, mientras que en España la cifra era del 43,1\% ${ }^{1}$. A inicios de 2001, Cataluña contaba con el 22\% de las líneas RDSI existentes en España. La Telefonía Rural de Acceso Celular (TRAC) aportaba a Cataluña una cifra simbólica de 12.662 líneas ${ }^{2}$.

\footnotetext{
${ }^{1}$ Estos datos sobre líneas de telefonía básica no incluyen el servicio Ibercom (servicio de centralitas privadas de Telefónica), ni RDSI (Red Digital de Servicios Integrados), ni ADSL (Línea de Abonado Digital Asimétrica).

2 Las fuentes más fiables para conseguir estos datos son los operadors, aunque, por razones de estrategia comercial, acostumbran a ser reacios a la hora de publicitar estadísticas sobre kilómetros de red extendida y cantidad de abonados. La Secretaria de Telecomunicacions i Societat de la Informació del DURSI (Generalitat de Cataluña) publica anualmente las Estadístiques de la Societat de la Informació-Catalunya, estudio elaborado por el Observatori de la Societat de la Informació a partir de datos del Institut d'Estadística de Catalunya (IDESCAT) y de otros organismos y fuentes oficiales. El objetivo consiste
} 
En este contexto, el cable, a finales de 2001, contaba con 37.064 abonados, es a decir, el 1,8\% del total de hogares de Menta, la denominación comercial de Cable i Televisió de Catalunya (CTC). Menta, cuyas previsiones de expansión de red se habían visto amortiguadas (CTC, 1996), acumulaba a inicios de 2002 una cifra de hogares cableados (aquellos que aún no tienen instalación dentro del edificio pese a que les llega el cable hasta la calle) que suponía el $16,5 \%$ de los hogares catalanes. A finales de 2002 la cifra de hogares cableados se aproximaba a los 500.000. Aproximadamente el $90 \%$ de esos hogares pueden contratar los servicios del cable (telefonía, televisión e Internet, el denominado triple play, ya que permite recibir la tríada de servicios gracias a la misma infraestructura), porque los tres llegan hasta la vivienda. Nos hallamos, pues, ante hogares con unidad activada, lo que significa que pueden abonarse al servicio cuando lo deseen. La red de Menta era a inicios de 2003 de más de 500 kilómetros de red desplegada, es decir, de kilómetros de zanja construidos, no de fibra óptica (Miller y Chynoweth, 1979; Tingye, 1985; Agrawall, 1992) o cable instalados (Baldwin y McVoy, 1988).

Los incrementos más espectaculares se están dando en la tecnología ADSL, que permite una conexión permanente a Internet con unas velocidades que oscilan entre los $256 \mathrm{Kbps}$ (kilobits por segundo) y los $2 \mathrm{Mbps}$ (megabits por segundo) a través del par de cobre de la línea telefónica convencional. Para Telefónica ( $y$, en general, para todos los antiguos monopolios de telefonía y telecomunicaciones de los diversos países en proceso de liberalización del mercado) se trata de una tecnología muy rentabilizadora, ya que explota al máximo la red de la que ya dispone y le libra de afrontar las costosas y lentas instalaciones de redes de cable, pese a que éstas disponen de más banda ancha y más posibilidades a corto plazo, sobre todo las fundamentadas en fibra óptica. Las centrales telefónicas equipadas con ADSL daban servicio a más del $94 \%$ de la población catalana en 2002, aunque, de las líneas ADSL en servicio, un $45 \%$ estaban contratadas por abonados particulares, y un $55 \%$ por

en dar a conocer los indicadores que miden la situación de la implantación de las tecnologías de la información y la comunicación (TIC) en Cataluña. 
empresas. La penetración del ADSL era de 0,87 líneas por cada 100 habitantes. Los hogares catalanes con acceso a Internet a alta velocidad en 2002 suponían poco más del $2,1 \%$.

Por tanto, uno de los retos de los operadores de cable era prever y luchar contra el previsible ascenso del ADSL. De hecho, la Audiencia Nacional denegó en diciembre de 2002 las medidas cautelares que solicitaban desde abril del mismo año Madritel, Menta, Cabletelca y otras empresas pertenecientes a Auna Cable (que agrupaba a los operadores de cable dominados por Endesa, Fenosa y SCH, es decir, Madritel, Cable de Aragón, Canarias Telecom, Menta y Supercable Andalucía), contra la decisión del Gobierno de permitir a Telefónica utilizar la tecnología ADSL para ofrecer servicios de cable.

Técnicamente, la decisión del Gobierno consistía en transformar las licencias de Telefónica para operar en telecomunicaciones por cable en otros títulos habilitantes, más laxos en exigencias financieras y tecnológicas. Esa decisión convirtió las telecomunicaciones en Cataluña y en España en un verdadero totum revolutum que permitió a Telefónica desarrollar los servicios de telecomunicaciones que debían ser por cable, incluyendo la transmisión de canales de televisión (Chauvet, 1986), a través del ADSL. Ello ha generado dos efectos perniciosos para la banda ancha catalana y española: ha congelado las costosas inversiones en la red de cable por la inesperada competencia surgida y el súbito cambio de modelo de negocio que supone el ADSL, y ha retrasado la constitución de una red alternativa a la del incumbente, lo que perjudica la salud de la sociedad de la información catalana y española.

Telefónica ya había renunciado al despliegue de más red de fibra óptica en el año 2000, contraviniendo el espíritu de la Ley de 1995. La red de Telefónica estaba preparada en más del $80 \%$ para dar ADSL, pero sólo entre el $50 \%$ y el $60 \%$ de esta 
tecnología permite la difusión de la señal de televisión con calidad ${ }^{3}$. En 2003 se revisaban las obligaciones de los operadores de cable: Telefónica tenía comprometidos más de 90 millones de euros en avales para responder a esas obligaciones.

En mayo de 2002 el acceso a Internet en Cataluña era del 29,9\% (Generalitat de Cataluña, 2002). De hecho, la sociedad de la información se iba introduciendo en los hogares catalanes progresivamente, y el $52,5 \%$ de las viviendas con acceso a Internet había contratado una tarifa plana, el $20,8 \%$ de los hogares estaban subscritos a servicios de televisión de pago (un $11,4 \%$ de ellos a televisión digital vía satélite) y más de $34 \%$ de catalanes utilizaban el correo electrónico para comunicarse con familiares y amigos.

La previsible fusión entre los dos grandes grupos de operadores de cable españoles (Ono y Auna Cable) se configuraba como la solución para constituir una alternativa sólida y estatal a Telefónica. Eso garantizaría más vías de acceso de la ciudadanía a la red de banda ancha. Si en la evolución de ese período analizamos como variable la coyuntura económica que afecta al sector telecomunicativo, el gap con relación a los países más desarrollados tecnológicamente crece. Las consecuencias son incalculables.

\subsection{Obstáculos a superar para el aumento de la penetración del} cable

La experiencia de los segundos operadores de cable demuestra que persisten y se agravan los males endémicos de la escasa duración de la moratoria (24 meses, tras

\footnotetext{
${ }^{3}$ De hecho, el ex monopolio ya era la segunda compañía entre las cinco grandes europeas con mayor porcentaje de líneas ADSL sobre el total de conexiones telefónicas. Deutsche Telekom era líder con el 4,3\%, y Telefónica segunda con el 2,2\% . A continuación se situaban Telecom Italia (1,5\%), France Télécom $(1,2 \%)$ y BT $(0,5 \%)$. La operadora española acabó 2001 con más de 375.000 conexiones de ADSL en España, sumando la oferta mayorista con la minorista, y las cifras de Terra y de Telefónica Data. El punto de equilibrio del negocio estaba fijado en el millón de líneas.
} 
los diversos cambios legislativos experimentados por esa cifra) y que aparecen algunos nuevos.

En primer lugar, hallamos complejidad burocrática y retrasos en la actuación de las administraciones públicas, sobre todo los ayuntamientos, para otorgar las concesiones de uso del dominio público municipal y de las licencias de obras. Ambas son imprescindibles para la creación de las infraestructuras necesarias para la prestación de servicios. La administración municipal ha percibido en la red una oportunidad de ingresos, cuando con una visión a largo plazo, en el entorno de la sociedad digital, la urgencia consiste en extender el máximo posible el acceso a la banda ancha.

En segundo lugar, los operadores de telecomunicaciones por cable topan con una clara dificultad de acceso a las infraestructuras de obra civil y a los servicios de Telefónica como operador dominante sin que existan aún alternativas suficientes. Ello convierte en más costoso el despliegue de la red de operadores entrantes (los new entrants). El entorno es de inseguridad en las negociaciones de interconexión con Telefónica y lentitud y escollos en su inicio y desarrollo. Es difícil de prever razonablemente la conclusión de los tratos, ya que el incumbente apuesta por una política de semibloqueo y de dilación a la hora de cerrar acuerdos.

En tercer lugar, persiste el desequilibrio tarifario. La rebaja artificial de las tarifas de llamadas locales perjudica a los operadores de cable entrantes, que deben competir en ese mercado y, especialmente, en el bucle de abonado, el verdadero aspecto clave para conseguir un régimen de libre competencia.

De cara al usuario, y como cuarto foco de posible mejora, se detecta una dificultad creciente de acceso a los contenidos. Este fenómeno se ha agravado a raíz de la fusión de las plataformas de televisión digital por satélite. Las exigencias para los operadores de telecomunicaciones por cable, que además deben competir en el dúo que completa 
el triple play (telefonía e Internet), pueden ser difícilmente soportables. A este hecho se añade que la mayor parte de competidores por los derechos de televisión han irrumpido en el mercado antes que los operadors de cable, de forma que éstos ya no disponen de los contenidos más atractivos para la audiencia.

La quinta barrera a superar en aras de conseguir una competencia satisfatoria para todos los elementos de la cadena de valor es la actividad comercial agresiva de Telefónica para anticipar la presencia en el mercado relevante de los servicios de telecomunicaciones por cable. El ex monopolio dibuja la estrategia de adelantarse al final de la moratoria: la adopción de la tecnología ADSL es un exponente de ello.

Finalmente, y como consecuencia de las dilaciones provocadas por el conjunto de factores anteriores, los operadores observan con preocupación la aparición de tecnologías diferentes al cable, com el LMDS (Local Multipoint Distribution System) o el MMDS (Multichannel Multipoint Distribution System), sistemas de comunicación punto multipunto que posibilitan la existencia del bucle local sin hilos. La lentitud en la extensión de la red de telecomunicaciones por cable favorece las expectativas de estas alternativas, concebidas especialmente para el ámbito rural, donde se retrasará más la llegada de las redes de banda ancha.

Estos escollos delatan el discutible cumplimiento de compromisos de los actores implicados en un sector capital para el desarrollo de un país (De Fontenay, Shugard y Sibley, 1990; Curwen, 1995). La extensión de las redes de telecomunicaciones por cable y de banda ancha en España y en Cataluña ha comenzado con retraso con relación a otros países de la Unión Europea, pero una reacción colectiva puede permitir adaptar el desarrollo de la infraestructura y del servicio a la normativa europea, que aboga por la liberalización, la armonización y la libre competencia (Baltz et altri, 1985; Benzoni y Hausman, 1993; Biole et altri, 1993; Bangemann, 1994, 1996; Alabau, 1998). 


\section{El caso de Cataluña: la transición para el cable histórico}

\subsection{El peso cuantitativo del cable local}

Las nebulosas que entorpecen la implementación de redes de los grandes operadores se convierten en nubarrones para los operadores de cable histórico, cuyo papel en España ha sido relevante sobre todo desde los años 70 del siglo XX (Ezcurra, 1973). Estas microempresas de la información se prodigaron en el arco mediterráneo. Una de las zonas con mayor densidad de pequeños operadores de cable es Cataluña (Gifreu, Recoder-Sellarès y Corbella, 1988), que sirve metodológicamente de estudio de caso para ilustrar una situación legal que tratan de afrontar de la forma más eficiente posible.

Así, para la administración pública catalana es fundamental la extensión acelerada de infraestructura, de red de banda ancha. La fibra óptica es el objetivo a largo plazo si tenemos en cuenta las previsiones de exponencialidad de acceso a la red (Gilder, 2002). Cataluña afrontó el cambio de siglo con signos de mejora y un reflejo del impacto de la digitalización en los pilares del Estado del bienestar: más del 50\% de los trabajadores de la Administración pública utilizaban ordenador y un $20 \%$ Internet; el $17,5 \%$ de los usuarios habituales de Internet visitaban páginas web sanitarias; los centros educativos de Cataluña disponían de una ratio de 12 alumnos por ordenador; los titulados en carreras TIC habían aumentado un 22,5\% en un año; la facturación de Cataluña en fabricación de equipos electrónicos y de telecomunicación en 2000 representaba el 36,8\% del total estatal; y el gasto global en I+D en Cataluña en 2000 fue el 1,11\% del PIB.

Un aspecto cualitativamente interesante es la mejora de la cohesión social con relación a las TIC. La extensión de la red de banda ancha hasta zonas rurales o de difícil acceso, mediante red física o por ondas electromagnéticas (LMDS o MMDS), puede confirmar la tendencia. Curiosamente, el uso de las TIC es similar en áreas 
rurales y en las metropolitanas. En las poblaciones de menos de 10.000 habitantes, utiliza ordenador el $52 \%$ de la población con 15 años o más, en municipios entre 10.000 y 500.000 habitantes lo hace el $59,6 \%$, y en Barcelona ciudad, el $57 \%$.

Estratégicamente, el acceso a la banda ancha en Cataluña se catalizaba mediante los operadores de cable histórico y los grandes operadores de la demarcación salidos del concurso (CTC o Menta y Telefónica). Un posible objetivo de políticas públicas consistía en establecer un operador propio, similar a Euskaltel en Euskadi. El ente que más se asemejaba y que estaba ayudando a configurar el espacio catalán telecomunicativo, aunque afectado por una crisis constante, era Al-pi, operador catalán para empresas (un operador de nicho, es decir, especializado), creado de la privatización de Catalana de Telecomunicacions, empresa segregada del Centre de Telecomunicacions de la Generalitat. Uni2 invirtió más de 78,13 millones de euros en la adquisición. El consorcio Localret, que agrupaba a 784 ayuntamientos catalanes, dio apoyo a la propuesta de la Generalitat para recomprar la compañía. En todo caso, habría que disociar la infraestructura de titularidad pública y la gestión, que debería continuar en manos de Uni2 o traspasarse a otro operador privado.

Más allá de los kilómetros de red HFC (Hibryd Fiber Coaxial, red híbrida de fibra óptica y cable coaxial) extendida por operadores como Telefónica o Menta (dentro de Aunacable), y la centrada en los rentables polígonos industriales (casos de Jazztel, Colt Telecom, al-pi u otros menos exitosos como Flash 10), Cataluña contaba con la sólida realidad de las redes de cable histórico.

El impacto del cable local (también se denomina así al cable histórico), nacido en época de alegalidad, es sensible en Cataluña y en España, casi siempre vinculado a experiencias de televisión local (Brenner y Price, 1986; Collins, 1992, 1994). Un ejemplo son los censos de Egeda (Entidad de Gestión de Derechos de los Productores Audiovisuales), que representa y defiende los intereses de los productores, derivados de los derechos reconocidos y protegidos por la Ley de Propiedad Intelectual. 
Asociaciones como Aesdica (Asociación Española de Servicios Distribuidos por Cable), que aglutina a 29 pequeños operadores, Acamur (Asociación de Operadores de Cable de Murcia), con 21 operadores y empresas de televisión local, Espacable (Asociación Española de Empresarios de Televisión por Cable y Televisión Local), con 174 emisoras, Local Media (más de 50 televisiones locales vía hertziana) y Acutel (Asociación de Televisiones por Cable de Andalucía), con más de 40 canales, reflejan el peso del fenómeno (Egeda, 1999). Las zonas con más operadores de cable histórico (Fondevila Gascón, 2004a) son Andalucía, donde se contabilizaban 253 redes, distribuidas en Sevilla (72 redes), Córdoba y Huelva (34), Granada (33), Jaén y Málaga (28) y Cádiz (24). Cataluña concentra 60 redes, Murcia 54 y Alicante, 28. Procono era la empresa de cable más implantada, ya que disponía de 60.000 suscriptores en 1990, cifra que descendería a 35.000 en 1994 y que en 2002 se mantenía alrededor de los 30.000 (Fondevila Gascón, 2003).

En ese entramado cuantitativamente tan relevante (y cualitativamente también desde el punto de vista comunicológico) destacan las redes de cable histórico catalanas, la mayoría dotadas de tecnología modesta. Así, la red extendida era de cable coaxial, que concede una escasa capacidad de canales en comparación con las redes híbridas y sobre todo con las redes de fibra óptica. Estas microempresas aparecieron en primer lugar por la necesidad de una correcta recepción de las ondas hertzianas. Como argumentos complementarios, las empresas de los municipios involucrados destacaban el incremento de la oferta de canales y el hecho de convertirse en una fórmula de expresión de la identidad local.

Si nos remontamos a la década de los años 80 del siglo XX, el cable local satisfacía funciones de teledistribución para mejorar la recepción de los canales de televisión existentes a raíz de las dificultades orográficas o climáticas. Otro motivo era estético; de hecho, ese argumento sobresalía en el caso de las casas sobre el río Onyar de Girona o en proyectos concretos, como la remodelación de la Plaça Major de Vic. Algunos municipios costeros o de montaña apelaban a intereses turísticos, puesto que 
la incorporación de una antena parabólica permitía captar numerosos canales extranjeros, lo que actuaba como aliciente para los visitantes y se traducía en más ingresos para el municipio. Esas ventajas eran secundadas por la mayor parte de operadores de cable histórico españoles.

La primera red de cable coaxial se comienza a instalar en Molins de Rei en 1966. La multiplicación se produjo en las décadas de los 70 y los 80 . Las redes observaban que el cliente necesitaba un servicio catalogable de básico (el televisivo), y que era fácilmente fidelizable. El pago por un servicio audiovisual se asentaba en el imaginario colectivo. Con todo, se ha considerado al vídeo comunitario (predecesor de la Community Antenna Television o CATV y de las redes de cable histórico) como una oportunidad histórica desaprovechada para impulsar el cable en España a causa de la inversión necesaria en infraestructuras y el presupuesto doméstico para la televisión (Álvarez Monzoncillo, 1997).

\subsection{Los condicionantes legislativos del cable histórico}

La única norma que regulaba los servicios de telecomunicaciones en los años 80 era la Ley 31/1987, de 18 de diciembre, de Ordenación de las Telecomunicaciones (LOT), que contemplaba la actividad del vídeo comunitario, precursor de la televisión por cable en España. La regulación presentaba restricciones, ya que sólo permitía la actividad si se operaba en un edificio sin ocupar la vía pública. Si estos vídeos comunitarios ocupaban el dominio público, se considerarían televisión por cable. Al ser este servicio de titularidad estatal, se requería una concesión previa, que era imposible de conseguir ante la carencia de una regulación específica (Salgado, 1995).

El Tribunal Constitucional (TC) se manifestaba contrario a los proyectos empresariales de televisión por cable, concebida como servicio público. Como tal, se debía someter a una regulación del procedimiento de adjudicación de las concesiones para garantizar 
los principios de libertad, igualdad y pluralismo político. En la sentencia $189 / 91^{4}$, el TC consideraba lícita la faculdad del legislador de declarar el servicio público de televisión sobre la base de la ocupación del dominio público. Las actividades de transmisión de señales que ocupasen más de un edificio serían consideradas servicio público.

Esta postura del TC no experimentaría cambios hasta la sentencia $31 / 94^{5}$, en la que permitía a las emisoras de televisión local proseguir con su actividad a raíz del vacío legal sobre la televisión por cable. Cabe tener en cuenta que se producía la imposibilidad práctica de obtener una concesión administrativa de emisión. Además, se recogía el espíritu del derecho a la libertad de expresión y comunicación garantizado por el artículo 20.1 de la Constitución.

En el ámbito político, en 1994 los ayuntamientos gobernados por el Partido Popular (PP) comenzaron a convocar concursos para otorgar títulos habilitantes a empresas de televisión por cable que operasen en el ámbito municipal. En paralelo, la Unión Europea aprobó la Directiva 95/51/CE, según la cual los Estados miembros deberían desarrollar un marco normativo para suprimir las restricciones al suministro de capacidad de transmisión a través de redes de cable y deberían autorizar su uso para la prestación de servicios de telecomunicaciones diferentes de los de telefonía vocal. La Resolución de 23 de mayo de 1996 ampliaba el plazo para resolver las concesiones provisionales para la explotación del servicio de telecomunicaciones por cable, previsto en la disposición transitoria 1a de la Ley 42/1995. Esta disposición establecía que las redes de televisión por cable que se encontrasen en explotación comercial a la entrada en vigor de la Ley 42/1995 podrían continuar con la explotación. Como esta condición tenía que ser demostrada, se debía solicitar una inspección al Ministerio de Obras Públicas, y se establecían unos plazos máximos para regularizar la situación. La decisión era esencial para los operadores de cable histórico, en situación de cierta indefinición que se arrastraría hasta años más tarde.

\footnotetext{
${ }^{4}$ STC 189/91 (vídeo comunitario Huelva).

${ }^{5}$ SDTC 31/94 (Lady Cocinas).
} 
Sea como fuere, el cable histórico recuperaba protagonismo en un texto legal. Ello reflejaba el peso de estas iniciativas de la sociedad civil y de los empresarios locales. La disposición transitoria primera establecía que las empresas adjudicatarias de concursos convocados por ayuntamientos para la instalación y explotación del cable antes de la entrada en vigor de la Ley 6/1996 y que no se hallasen en explotación comercial el 24 de diciembre de 1995 debían participar en el primer concurso convocado por el Ministerio de Fomento, en los términos regulados en la Ley 42/1995, para la adjudicación de la correspondiente concesión. La no participación en el concurso se entendía como renuncia a los derechos derivados de la disposición ${ }^{6}$.

Los operadores de cable local obtuvieron una concesión provisional para seguir operando en su ámbito siempre que los operadores con concesión administrativa no comercializasen la oferta en el ámbito en cuestión (Ley 50/1998, de 30 de diciembre, de Medidas Fiscales, Administrativas y de Orden Social). Sin embargo, la Ley General de Telecomunicaciones de 1998 modificó el escenario, ya que cualquier operador podía solicitar licencias para ofrecer el servicio de telefonía e Internet, y no de televisión. Los operadores de cable histórico podían arrendar su red para servicios de telefonía e Internet.

A raíz de la legislación del sector, algunas empresas del cable histórico y otras del sector multimedia y afines se integraron en el accionariado de algunos operadores. Emulaban así la senda tomada por la mayor parte de los medios de comunicación y del sector editorial y audiovisual.

\footnotetext{
${ }^{6}$ Si las empresas o entidades no resultaban adjudicatarias en el concurso, tendrían derecho a obtener una concesión especial y no renovable para la prestación del servicio de televisión por cable en un plazo de diez años a partir del plazo fijado para completar la instalación de la red, determinado atendiendo la inversión prevista y otras circunstancias. Si no se participaba en el concurso, se entendía que los interesados renunciaban a los derechos señalados en la disposición transitoria. Los titulares de redes incluidos en el régimen de la disposición transitoria primera de la Ley 42/1995 también podían solicitar al Ministerio de Fomento, en el plazo de dos meses desde la entrada en vigor de la ley, acogerse a estos beneficios sin que se precisara autorización para realizar inversiones en la red que explotaban.
} 
El recorrido de futuro del cable generó algunos proyectos curiosos. En los años 80, el Ayuntamiento de L'Hospitalet de Llobregat solicitó a Telefónica un estudio para cablear toda la ciudad para recibir la emisión de los canales de televisión sintonizables en Cataluña, crear un canal local y reservar el resto para los otros servicios. La dirección general de Arquitectura i Habitatge de la Generalitat de Cataluña, a través del Departament del Patrimoni Artístic, intentó cablear los lugares de interés artístico con el objetivo de favorecer a los municipios en cuestión. La misión era eliminar las antenas de televisión, como en los primeros documentos justificadores del cable en España, a inicios de los años 70 . El sistema de cableado se destinaba a la recepción de televisión y frecuencia modulada.

Durante los años 90, Cataluña acogía unas 60 redes de cable local. Su representatividad era elevada, ya que en 24 de las 41 comarcas catalanas existía alguna red. Destaca el Berguedà (11 redes: Avià, Bagà, Berga, Borredà, Castellar de n'Hug, Cercs, Gironella, Guardiola de Berguedà, La Pobla de Lillet, Puig-reig y Vilada), seguida por el Bages ( 9 redes: Avinyó, Balsareny, Cardona, Monistrol de Montserrat, El Pont de Vilomara, Sallent, Sant Feliu Sasserra, Santa Maria d'Oló y Súria), Anoia (7 redes: Capellades, Carme, Igualada, La Llacuna, Piera, La Pobla de Claramunt y Sant Martí de Tous), Alt Penedès ( 5 redes: Gelida, Lavern, Mediona, Pontons y Vilafranca del Penedès), Baix Llobregat (3: Castellví de Rosanes, Gavà y Molins de Rei), Alt Empordà (3: Cadaquès, L'Escala y Peralada), Ribera d'Ebre (2: Ascó y Riba-roja d'Ebre), Maresme (Caldes d'Estrac y Sant Pol de Mar), Montsià (Alcanar y La Sènia), Garrotxa (Castellfollit de la Roca y Sant Feliu de Pallerols), Ripollès (Camprodon), Conca de Barberà (Conesa), Priorat (Cornudella de Montsant), Vallès Oriental (Gualba), Baix Empordà (Pals), Baix Ebre (El Perelló), Alta Ribagorça (El Pont de Suert), Cerdanya (Puigcerdà), Segarra (Sanaüja), Osona (Sant Bartomeu del Grau), Solsonès (Solsona), Urgell (Vallbona de les Monges), Baix Penedès (Vendrell) y Baix Camp (Vinebre).

Por provincias, Barcelona (sobre todo las comarcas interiores), con 39 empresas de cable local, concentra un número operadores mayor que Tarragona (9), Girona (8) y Lleida (4). 
La media de población cubierta por los operadores de la época de alegalidad está por debajo de los cinco mil habitantes. Por tanto, el carácter de estas redes es prioritariamente rural y potencialmente redistribuidor desde una perspectiva territorial. En cuanto a cantidad de abonados, la cifra global roza los veinte mil (19.611), pero, cruzada con la cantidad de viviendas pasadas (a las que llega la red: 55.431), arroja un $35,38 \%$ de éxito. Ese porcentaje es muy elevado, ya que las tasas de los grandes operadores internacionales (CIT, 2000) raramente llegan al 30\% de hogares abonados sobre el total de hogares pasados (Fondevila Gascón, 2004b). La respuesta de la sociedad civil se revela crucial. La media de abonados por operador es de 384,52, y la de viviendas pasadas, de 1.179,38. La distribución por propiedad de los operadores demuestra una primacía de los privados (39, propiedad de 10 operadores) ante los públicos (15) y los mixtos (1). Los privados acostumbran a ser empresas de volumen medio. En ocasiones, los operadores sólo se encargan de la instalación, y después las redes pasan a depender de los ayuntamientos locales o se convierten en mixtas.

Las tendencias de los operadores de cable locales pueden variar en función de su ubicación. En algunos casos, lo previsble es una aproximación al operador ganador del concurso de las demarcaciones y un traspaso tanto de la red como de la cartera de clientes, especialmente fieles a unas empresas que les han aportado la señal televisiva durante décadas. En otros casos, sobre todo aquellos en los que los grandes operadores, por razones de negocio, no manifiestan la intención de llegar con su red, el operador históricvo podrá continuar su tarea sine die. Al tratarse de municipios con carga demográfica modesta, los grandes operadores tampoco encontrarán a faltar esa simbólica cantidad de abonados. Otro escenario es que el pequeño operador continúe funcionando (sobre todo en capitales de comarca o municipios de mayor envergadura y posibilidades de facturación), aun a sabiendas de que el gran operador llegará tarde 0 temprano. En ese supuesto, cuando el despliegue de la red del gran operador sea inminente, se puede optar por la opción del traspaso o por la disolución de la empresa. En todo caso, sea una u otra opción, en general la vetusta red de cable coaxial ya habrá sido amortizada con creces, por lo que desde una perspectiva economicista se 
habrá tratado de un negocio redondo dentro de su relativa modestia. Teniendo en cuenta las estrecheces presupuestarias y financieras, la sencillez de las instalaciones técnicas, los costos de explotación y el carácter voluntarista de alguna de las aventuras de cable local, los resultados son considerables. Además, el pausado (en muchas ocasiones por razones exógenas) ritmo de cableado por parte de los grandes operadores y la política pasiva y rentabilizadora de las redes de par de cobre tradicionales mediante la tecnología ADSL por parte de Telefónica benefician la opción de prolongación de la actividad del cable tradicional.

Los grandes operadores argumentan que la opción de la absorción no es óptima en muchos casos, ya que la adaptabilidad técnica de algunas de las redes (sobre todo las primigenias) no es siempre factible. La adaptación a la red HFC puede implicar prescindir en parte 0 en la totalidad de la red de cable coaxial y no poder ofrecer la integración de servicios (televisión, telefonía, Internet), aunque se valora la cartera de clientes que se pueda traspasar.

\section{Conclusión}

El fenómeno de los operadores de cable histórico en España ha dinamizado el panorama audiovisual español en una época de monolitismo legislativo en contraste con la vocación de pluralidad manifestada por la sociedad civil, especialmente la de los municipios afectados. El mecanismo bottom up, es decir, la búsqueda de soluciones y el activismo procedente de la ciudadanía ante la parálisis y el retraso del legislador y el deseo de recibir correctamente y con más posibilidades el servicio televisivo, demuestra que en sectores tan estratégicos como el comunicativo y el telecomunicativo la capacidad de control absoluto no lo abarca todo. Fenómenos como la popularización de Internet pueden ahondar en ese camino.

Tras las modificaciones legislativas, se abren diversas vías de futuro para las empresas aquí analizadas. Sea en una dirección o en otra, las redes de cable histórico han 
demostrado fortaleza y han ofrecido un servicio esencial para los municipios en los que se han implantado. La interpretación comunicológica de la realidad local no es posible, por tanto, sin analizar un fenómeno singular que se ha prolongado y se prolongará en el tiempo.

\section{Bibliografía}

AGRAWALL, Govind P. (1992). Fiber-Optic Communications Systems. John Wiley \& Sons, Inc. New York.

ALABAU, Antonio (1998). La Unión Europea y su política de telecomunicaciones. En el camino hacia la Sociedad de la Información. Fundación Airtel Móvil. Madrid.

ÁLVAREZ MONZONZI LLO, J osé María (1997). Imágenes de pago. Fragua. Madrid.

BALDWIN, Thomas F., y MCVOY, D. Stevens (1988). Cable Communication. PrenticeHall Inc. Englewood Cliffs.

BALTZ, Claude; DUBOIS, Gérard; LE PERS, Marc, y THONON, Marie (1985). Les stratégies européennes de telédistribution. Centre Pompidou. Paris.

BANGEMANN, Martin (1994). "Europa y la sociedad global de la información: recomendaciones del grupo de alto nivel sobre la sociedad de la información al Consejo Europeo de Corfú" (pp. 5-40), Boletín de la Unión Europea (suplemento).

BANGEMANN, Martin (1996). "El desafío de la competitividad". En La Europa sin fronteras, Dirección General "Información, Comunicación, Cultura y Sector Audiovisual", Comisión Europea, n. 5, mayo 1996. 
BENZONI, Laurent, y HAUSMAN, Jerry (1993). Innovation, déréglamentation et concurrence dans des télécommunications. Eyrolles. Paris.

BIOLE, Alain; DE LA TORRE, Luís; LAURAIRE, Richard, y NÉGRIER, Emmanuel (1993). Les politiques publiques de télécommunication en Europe du sud. Service public et dynamiques territoriales des intérêts. CEPEL, Université de Montpelier. Montpelier.

BRENNER, Daniel L., y PRICE, Monroe E. (1986). Cable Television and Other Nonbroadcast Video: Law and Policy. Clark Boardman Company. New York.

BUSTAMANTE, Enrique (1999). La televisión económica. Financiación, estrategias y mercados. Gedisa. Barcelona.

CALVO CHARRO, María (1997). La televisión por cable. Marcial Pons. Madrid.

CASTELLS, Manuel (1997). La era de la información: Economía, sociedad y cultura. Volumen 1: La sociedad red. Alianza Editorial. Madrid.

CASTILLA, Adolfo (ed.) (1989). La economía de las telecomunicaciones, la información y los medios de comunicación. Fundesco. Madrid.

CIT (2000). European Cable Systems Survey 2000. CIT Group Company. London.

COLLINS, Richard (1992). Satellite Television in Western Europe. J ohn Libbey. London.

COLLINS, Richard (1994). Broadcasting and audio-visual policy in the European single market. John Libbey. London. 
COMISIÓN EUROPEA (1994). Crecimiento, competitividad, empleo. Retos y pistas para entrar en el siglo XXI. Libro Blanco, Oficina de Publicaciones Oficiales de las Comunidades Europeas. Luxemburgo.

CHAUVET, Philippe (dir.) (1986). Le cable. La télévision au pluriel. Enterprise Moderne d'Edition. Paris.

CTC (Cable i Televisió de Catalunya, SA) (1996). Telecomunicacions per cable. Una eina per al desenvolupament econòmic social. CTC. Barcelona.

CURWEN, Peter (1995). "Telecommunications policy in the European Union: developing the information superhighway", en Journal of Common Market Studies, vol. 33. Pág. 331-360.

DE FONTENAY, Alain; SHUGARD, Mary H., y SIBLEY, David S. (1990). Telecommunications demand. Modelling an integrated view. North-Holland. Amsterdam.

EGEDA (Entidad de Gestión de Derechos de los Productores Audiovisuales) (1999). Informe anual. EGEDA. Madrid.

EZCURRA, Luis (1973. "La télévision par câble, realité inmediate en Espagne", en Revue de I'UER, vol. 24: 1. Pág. 44-50.

FARIAS, Pedro, y MULTIGNER, Gilles (eds.) (1995). La televisión local ante el reto del cable. Diputación Provincial de Zaragoza, Servicio de Publicaciones. Zaragoza.

FONDEVILA GASCÓN, Joan Francesc (2003). El Cable en España, 2002. CECABLE. Terrassa. 
FONDEVILA GASCÓN, Joan Francesc (2004a). El Cable a Catalunya, 2003. CECABLE. Terrassa.

FONDEVILA GASCÓN, Joan Francesc. (2004b). Cable in Spain, 2003. CECABLE. Terrassa.

GARRIGA, Antoni M. (1985). "L'experiència de la televisió en l'àmbit mesocomunicatiu (Bases per a una televisió local a Espanya)" (pp. 49-80). 1eres Jornades sobre Mesocomunicació a Catalunya. Universitat Autònoma de Barcelona (UAB). Bellaterra.

GATES, Bill (1995). Camino al futuro. McGraw Hill. México.

GENERALITAT DE CATALUÑA (2002). Enquesta sobre la penetració de les Tecnologies de la Informació i la Comunicació a la societat catalana. STSI IDESCAT. Barcelona.

GIFREU, J osep; RECODER-SELLARÈS, M. Josep, y CORBELLA, Joan M. (1988). La comunicació per cable a Catalunya. Institut d'Estudis Catalans. Barcelona.

GILDER, George (2002). Telecosm: The World After Bandwidth Abundance. Touchstone. New York.

JUNTA DE CASTILLA Y LEÓN (1995). Televisión por cable y autopistas de la información. Consejería de Fomento de la J unta de Castilla y León. Valladolid.

LÓPEZ-ESCOBAR, Esteban, y BERTRAND, Jean-Claude (1986). La televisión por cable en América y Europa. Fundesco. Madrid. 
MARCUS, Norman (1986). Broadcast and cable management. Prentice-Hall. Englewood Cliffs.

MILLER, Stewart E., y CHYNOWETH, Alan G. (1979). Optical fiber telecommunications. Academic Press. New York.

MOSCO, Vincent, y WASKO, Janet (eds.) (1988). The Political Economy of Information. University of Wisconsin Press. Madison.

REGOURD, Serge (1992). La télévision des Europééns. Institut International d'Administrations Publiques. Paris.

SALGADO, Elena (1995). "La regulación del cable en España" (pp. 157-167). En FUNDESCO. Telecomunicaciones 1995. Tendencias. Fundesco. Madrid.

TINGYE, Li (dir.) (1985). Optical fiber communication (Fiber fabrication). Academic Press. Orlando. 